\title{
TRANSMEMBRANE ORIENTATION OF AN EARLY BIOSYNTHETIC FORM OF ACETYLCHOLINE RECEPTOR $\delta$ SUBUNIT DETERMINED BY PROTEOLYTIC DISSECTION IN CONJUNCTION WITH MONOCLONAL ANTIBODIES'
}

\author{
DAVID J. ANDERSON, ${ }^{*, 2}$ GÜNTER BLOBEL, ${ }^{*}$ SOCRATES TZARTOS,,${ }^{3}$ WILLIAM GULLICK,,+4 AND \\ JON LINDSTROM† \\ * Laboratory of Cell Biology, The Rockefeller University, New York, New York 10021 and $\ddagger$ The Salk Institute, \\ San Diego, California 92138
}

Received January 10, 1983; Accepted March 31, 1983

\begin{abstract}
The transmembrane topology of acetylcholine receptor (AChR) $\delta$ subunit, synthesized in vitro and co-translationally integrated into dog pancreas rough microsomal membranes, was studied using limited proteolysis and domain-specific immunoprecipitation. Forty-four kilodaltons (kd) of the $65-\mathrm{kd} \delta$ subunit comprise a single fragment that is inaccessible to exhaustive proteolytic digestion from the cytoplasmic surface of the membrane by trypsin, chymotrypsin, thermolysin, and pronase. Previously, we have shown that this 44-kd "protected" fragment contains the amino terminus of the intact molecule and all of the core oligosaccharides (Anderson, D. J., P. Walter, and G. Blobel (1982) J. Cell Biol. 93: 501-506). Here we demonstrate that this domain can be further dissected into a 26-kd fragment, together with low molecular weight material, when the membranes are rendered permeable to trypsin by low concentrations of deoxycholate (Kreibich, G., P. Debey, and D. D. Sabatini (1973) J. Cell Biol. 58: 436-462). This 26-kd fragment contains all of the core oligosaccharides present on the intact subunit and therefore constitutes at least part, if not all, of the extracellular domain. The remaining low molecular weight material may derive from the membrane-embedded domain; our data imply that as much as $18 \mathrm{kd}$ may be internal to the lipid bilayer. On the other hand, part of the cytoplasmic pole of AChR- $\delta$ can be recovered as a discrete, 12 -kd fragment upon mild trypsinization of intact vesicles. We have used this 12 -kd fragment to identify anti-AChR- $\delta$ monoclonal antibodies (mAbs) that react with the cytoplasmic domain of this subunit. Partial proteolytic fragmentation of the $\mathrm{AChR}$ in vitro translation products, in topologically well defined rough microsomes, may be used as a general assay to characterize the domain specificity of anti-AChR mAbs. For example, in the case of AChR- $\beta$, we were able to identify two mAbs that recognize extracellular and cytoplasmic fragments, respectively.
\end{abstract}

The acetylcholine receptor (AChR) of electroplax tissue has proven the most accessible model system for structural studies of a transmitter-activated ion channel. AChR isolated from Torpedo californica is a pentameric

\footnotetext{
${ }^{1}$ We thank Dr. Reid Gilmore for helpful discussions and a critical reading of the manuscript, Dr. Arthur Karlin for his comments on an early draft, and Drs. Toni Claudio and Steven Heinemann for making their results available to us prior to publication. Supported by a grant from the Muscular Dystrophy Foundation.

${ }^{2}$ To whom correspondence should be addressed, at Institute of Cancer Research, Columbia University, 701 West 168th Street, New York, NY 10032.

${ }^{3}$ Present address: Institut Pasteur Hellenique, 127 Avenue Vassilissis Sofias, Athens, Greece.

${ }^{4}$ Present address: Protein Chemistry Laboratory, Imperial Cancer Research Fund, Lincoln Inn Fields, London WC2A 3PX England.
}

complex of four polypeptides, $\alpha, \beta, \gamma$, and $\delta$, present in stoichiometry 2:1:1:1 (for review, see Karlin, 1981). These chains have molecular weights of $40(\alpha), 50(\beta)$, $60(\gamma)$, and $65(\delta)$ kilodaltons $(\mathrm{kd})$ and are tightly associated in a noncovalent complex (Weill et al., 1974; Reynolds and Karlin, 1978). The $\alpha$ subunit has been shown to contain the ACh-binding site (Karlin and Cowburn, 1973), and the $\delta$ subunit is labeled by the noncompetitive antagonist trimethisoquin (Oswald et al., 1980). However, the precise functional role of all four chains is unknown. The subunits surround a central depression (thought to be the ion channel), and their exact arrangement in this annular configuration has been proposed, based on electron microscopic (Wise et al., 1981) and xray diffraction (Kistler et al., 1982) data.

A major problem in AChR structure concerns the 
transmembrane topology of the subunits. In Torpedo plasma membrane vesicles, all four subunits are known to be exposed at the extracellular surface (Saint-John et al., 1982). Studies using proteolytic enzymes have suggested that each of the AChR chains communicates with the cytoplasmic compartment as well (Wennogle and Changeux, 1980; Strader and Raftery, 1980). However, these latter studies were compromised by the lack of a uniform, "inside-out" vesicle preparation and the consequential use of perturbative treatments, such as alkaline $\mathrm{pH}$ extraction or freeze-thaw, to expose the intracellular surface of the membrane.

Previously, we have used a cell-free protein-synthesizing system, supplemented with canine pancreas rough microsomes (RMs) (Blobel and Dobberstein, 1975) to study the transmembrane topology of early biosynthetic forms of the AChR subunits (Anderson and Blobel, 1981). As these microsomal membrane vesicles are topologically inverted with respect to the plasma membrane, this system permits unrestricted access of proteolytic enzymes to the cytoplasmic poles of the AChR chains. Earlier studies of membrane proteins, such as vesicular stomatitis virus $G$ protein, erythrocyte band III, and opsin, indicated that their transmembrane orientation in this in vitro system was indistinguishable from that of their authentic counterparts in homologous, native membranes (Katz et al., 1977; Goldman and Blobel, 1981; Braell and Lodish, 1982.) Indeed, in an earlier study (Anderson and Blobel, 1981), we obtained membrane-protected proteolytic fragments of 35 and $44 \mathrm{kd}$ for the $\alpha$ and $\delta$ subunits, respectively, and these data are in close accord with those obtained from $\mathrm{NaOH}$-extracted Torpedo vesicles (Wennogle and Changeux, 1980). In addition, we were able to define analogous membrane-protected tryptic fragments of 37 and $45 \mathrm{kd}$, for the $\beta$ and $\gamma$ subunits, respectively. These membraneprotected fragments contained all of the core oligosaccharides of, and, in the case of the $\delta$ subunit, the same amino terminal sequence as, the intact molecule (Anderson and Blobel, 1981; Anderson et al., 1982). Again, this result was consistent with that obtained for $\alpha$ and $\delta$ in the mature pentameric complex (Wennogle et al., 1981).

In this paper we have extended our studies of the transmembrane orientation of the $\delta$ subunit. The trypsin-resistant, 44-kd glycosylated domain is not further degraded from the cytoplasmic surface by chymotrypsin, thermolysin, and pronase. Exposure of this $44-\mathrm{kd}$ domain to trypsin, in vesicles made permeable by low concentrations of deoxycholate, converted it to a $26-\mathrm{kd}$ species. This 26-kd domain contained all the core oligosaccharide and must thus be extracellularly disposed. As this leaves almost $18 \mathrm{kd}$ of $\mathrm{AChR}-\delta$ which might be embedded in the lipid bilayer, this subunit is likely to contain more than one transmembrane segment.

We have also proteolytically dissected the AChR- $\delta$ cytoplasmic domain. Twelve kilodaltons of this domain can be immunoprecipitated as a discrete fragment when proteolysis is carried out with very low concentrations of trypsin. We have used this fragment in a novel assay to identify several monoclonal antibodies (mAbs) reactive with the cytoplasmic domain of AChR- $\delta$. We have extended this approach to the $\beta$ subunit and have identified
mAbs reacting with complementary cytoplasmic and membrane-protected proteolytic fragments of this chain.

\section{Materials and Methods}

Reagents. $\left[{ }^{35} \mathrm{~S}\right]$ Methionine (specific activity, $1000 \mathrm{Ci} /$ mmol) was obtained from New England Nuclear, Boston, MA. $\left[{ }^{3} \mathrm{H}\right]$ Leucine $(140 \mathrm{Ci} / \mathrm{mmol})$ was purchased from Amersham Corp., Arlington Heights, II. Protein A-Sepharose CL-4B was from Pharmacia, Uppsala, Sweden. Trasylol was from Mobay Chemical Corp., New York, NY; TPCK-trypsin was from Worthington Biochemical Corp., Freehold, NJ; thermolysin was from CalbiochemBehring, Inc.; and chymotrypsin $\mathrm{A}_{4}$ and pronase were from Boehringer Mannheim Biochemicals, Indianapolis, IN. Endo- $\beta-N$-acetylglucosaminidase $\mathrm{H}$ (Endo $\mathrm{H}$; EC 3.2.1.96) was the generous gift of Dr. Phillips Robbins, Massachusetts Institute of Technology, Cambridge, MA.

In vitro synthesis of $A C h R-\delta$ subunit. The isolation of Torpedo californica RNA, its translation in a micrococcal nuclease-digested wheat germ cell-free protein-synthesizing system and subsequent immunoprecipitation of AChR- $\delta$ using a rat polyclonal anti- $\delta$ antiserum were as described elsewhere (Anderson and Blobel, 1981). In some cases, immunoprecipitated translation products were treated with Endo $\mathrm{H}$ as previously described (Anderson et al., 1982).

Reisolation of rough microsomes from in vitro translation reaction mixtures. One-hundred-microliter translation reaction mixtures that had been incubated in the presence of $2 \mathrm{~A}_{280}$ units $/ \mathrm{ml}$ of $\mathrm{RM}$ were cooled on ice and supplemented with $21 \mu \mathrm{l}$ of $50 \mathrm{~A}_{280}$ units/ml of $\mathrm{RM}$ as carrier. These samples were next layered over a step gradient consisting of $25 \mu \mathrm{l}$ of $2.0 \mathrm{M}$ sucrose overlaid with $90 \mu \mathrm{l}$ of $0.5 \mathrm{M}$ sucrose in: $50 \mathrm{~mm}$ Tris- $\mathrm{HCl}, \mathrm{pH} 7.4,50$ $\mathrm{mm} \mathrm{KOAc}, 5 \mathrm{~mm} \mathrm{MgAc}(\mathrm{OAc})_{2}, 50 \mu \mathrm{g} / \mathrm{ml}$ of albumin and 100 units $/ \mathrm{ml}$ of Trasylol, in a nitrocellulose Airfuge tube. The gradients were centrifuged for $2 \mathrm{~min}$ at $100,000 \times$ $g_{\text {av }}$ in the $k=19$ rotor of the Airfuge ultracentrifuge (Beckman Instruments, Inc., Palo Alto, CA). Onehundred forty microliters of the supernatant were discarded, and the membranes were recovered from the 2.0 $\mathrm{M} / 0.5 \mathrm{M}$ sucrose interface in a volume of $25 \mu \mathrm{l}$ using a Hamilton syringe. When multiple samples were prepared in parallel, membranes were at this stage pooled and realiquotted to eliminate differences in recovery from the gradients.

\section{Results}

Dissection of the 44-kd glycoslyated domain. Previously, we had demonstrated that proteolysis of AChR- $\delta$ at its cytoplasmic pole, with high concentrations of trypsin, converted it to a single, glycosylated $44-\mathrm{kd}$ fragment that was protected by the membrane from further degradation (Anderson et al., 1982). Given the topology of the microsomal vesicles, this $44-\mathrm{kd}$ fragment must contain both extracellular and membrane-embedded domains. We therefore sought to dissect these two domains, to determine their relative sizes. First, we asked whether the 44kd segment could be further degraded by proteolysis from the cytoplasmic surface of the membrane, using proteases other than trypsin. Fxhaustive cleavage with thermolysin yielded the same, single $44-\mathrm{kd}$ protected fragment 
(Fig. $1 B$, lane 3) as that produced by either trypsin (Anderson and Blobel, 1981) or trypsin plus chymotrypsin (Fig. 1A, lane 1), albeit with slower kinetics (cf. Fig. $1 B$, lanes 1 and 3 ). The controls showed that no adventitious degradation occurred in the absence of protease despite the long incubation time (Fig. $1 B$, lanes 2 and 4), and that pre-AChR- $\delta$ (synthesized in the absence of RM) was under the same conditions completely degraded by thermolysin (not shown). Similarly, pronase also yielded a single membrane-protected fragment of $\mathrm{AChR}-\delta$ (Fig. $1 C$, lane 2), although it was slightly larger than that produced by trypsin (cf. Fig. $1 C$, lane 1 ).

Given that the 44-kd fragment is resistant to further cleavage from the cytoplasmic surface by a variety of proteases, we attempted to subfragment it into extracellular and membrane-embedded domains, by proteolytic attack from the opposite (i.e., luminal) side of the membrane. Since RMs are sealed, this experiment required conditions which rendered the vesicles permeable to exogenously added proteases, but which did not solubilize integral membrane proteins. Such conditions have previously been established for rat liver RMs using the detergent sodium deoxycholate (DOC) (Kreibich et al., 1973). We therefore established analogous conditions for the dog pancreas RMs used in this study.

At a membrane concentration of about $7 \mathrm{~A}_{280}$ units/ $\mathrm{ml}$, DOC concentrations of $0.5 \%$ or more essentially quantitatively solubilized AChR- $\delta$, as judged by a centrifugation assay (Fig. $2 A$, sup 0.5 ). At $0.2 \%$ DOC, only about $60 \%$ of the material was released from the membranes, and at $0.1 \%$ DOC, close to $90 \%$ of the AChR- $\delta$ was recovered in the pellet fraction (Fig. $2 A$, pellet, cf. 0 and 0.1 ). Nonetheless, $0.1 \%$ DOC rendered these RMs significantly permeable to externally added trypsin, as judged by the nearly complete degradation of entrapped bovine prolactin (Fig. $2 B$, compare 0 and $0.1 \%$ DOC). This concentration of detergent was chosen, therefore, to proteolyze AChR- $\delta$ simultaneously from the extracellular and cytoplasmic sides of the membrane.

Partial trypsinization of AChR- $\delta$ in the presence of DOC largely converted the $44-\mathrm{kd}$ protected fragment (cf. Fig. 1) to a 26-kd species (Fig. 3, cf. lanes 1 and 3, arrow) and, in addition, generated material in the molecular weight range of 10,000 to 15,000 (Fig. 3, open and solid arrowheads, lanes 3 and 4 ); the origin of some of these fragments will be discussed shortly. Digestion with Endo $\mathrm{H}$ indicated that the 26-kd fragment was glycosylated (Fig. 3, cf. lanes 3 and 4), and, based on the magnitude of the molecular weight shift produced by Endo H, contained all of the core oligosaccharides present in the parent 44-kd fragment (Fig. 3, cf. lanes 1 and 2 with 3 and 4). By these criteria, the 26 -kd fragment must contain an extracellular domain of AChR- $\delta$. (Closer examination reveals that the 26 -kd species and its $20-k d$ Fndo $\mathrm{H}$ digestion product are actually a series of bands, suggesting that this domain can be severed by trypsin at multiple cleavage sites in close proximity.) None of the additional low molecular weight bands (Fig. 3, lanes 3 and 4, arrowheads) was Endo $\mathrm{H}$ sensitive. One of them, in fact (Fig. 3, open arrowhead), derives from the cytoplasmic pole of AChR- $\delta$ (see below). The recovery of the 26 - $\mathrm{kd}$ material was consistently poor relative to the recovery of the parent 44-kd fragment. This is most likely due to the low titers of antibodies against this domain in the serum, as confirmed by Western blotting experiments using trypsinized mature AChR (not shown) and as reported previously by others (Froehner, 1981). It is also consistent with our failure to detect any mAbs reactive with this domain (see below.)

The observed subfragmentation of the 44-kd species to a 26-kd species in the presence of DOC was not due to a detergent-induced conformational change in the molecule. Pre-AChR- $\delta$, synthesized in the absence of microsomal membranes (Anderson et al., 1982), exhibited the same set of tryptic fragments (see Fig. 4, below) whether digested with or without DOC (data not shown). Thus, the appearance of new trypsin-sensitive sites on AChR- $\delta$ in the presence of DOC is most likely due to elimination of the membrane permeability barrier, and thence exposure of cleavage sites at the luminal surface, rather than to some other effect of detergent on the protease sensitivity of the molecule. Furthermore, the 26 -kd fragment was in fact generated from a membranebound form of AChR- $\delta$, as sedimentation of the DOCtreated microsomes, prior to proteolysis, yielded a supernatant fraction that produced virtually none of the 26 $\mathrm{kd}$ species upon subsequent trypsinization (not shown). The yield of this fragment from the DOC-permeabilized microsomes was not affected by increasing concentrations of detergent, but could be increased by raising the concentration of trypsin (data not shown).

Subfragmentation of the cytoplasmic domain of AChR$\delta$. As confirmed in the foregoing experiments (Fig. 1), almost $20 \mathrm{kd}$ of AChR- $\delta$ are accessible to proteolytic enzymes at the cytoplasmic surface of the rough endoplasmic reticulum membrane (Anderson and Blobel, 1981). However, these results were obtained in a limitdigestion experiment, designed to identify those domains of the subunit that were protected from proteolysis by the lipid bilayer. In order to examine the structure of the complementary, cytoplasmic domain, we required conditions for a limited proteolytic dissection of $\mathrm{AChR}-\delta$. We also needed to be certain that our antibodies would react with the fragments generated by such a dissection, once they were severed from the rest of the molecule.

To achieve these objectives, we used the precursor to AChR- $\delta$ (pre-AChR- $\delta$ ), synthesized in the absence of $\mathrm{RM}$, to explore the intrinsic sensitivity of the molecule to low concentrations of trypsin, without the interference of the membrane or of detergents. Our polyclonal antibody to AChR- $\delta$ had been prepared against sodium dodecyl sulfate (SDS)-denatured material; hence all immunoprecipitations could be performed after SDS dissociation of the trypsinized in vitro translation products. Partial trypsinization of pre-AChR- $\delta$ yielded a number of immunoprecipitable fragments (Fig. 4, lanes 50 and 250 ), including several in the molecular weight range of 12,000 to 20,000 expected for the putative cytoplasmic domain. A similar pattern of tryptic peptides was obtained when proteolysis was performed after immunoprecipitation or when $\left[{ }^{3} \mathrm{H}\right]$ leucine was used as the label; therefore, most of the tryptic fragments apparently were recovered by this procedure (data not shown.)

These mild trypsinization conditions were next used 


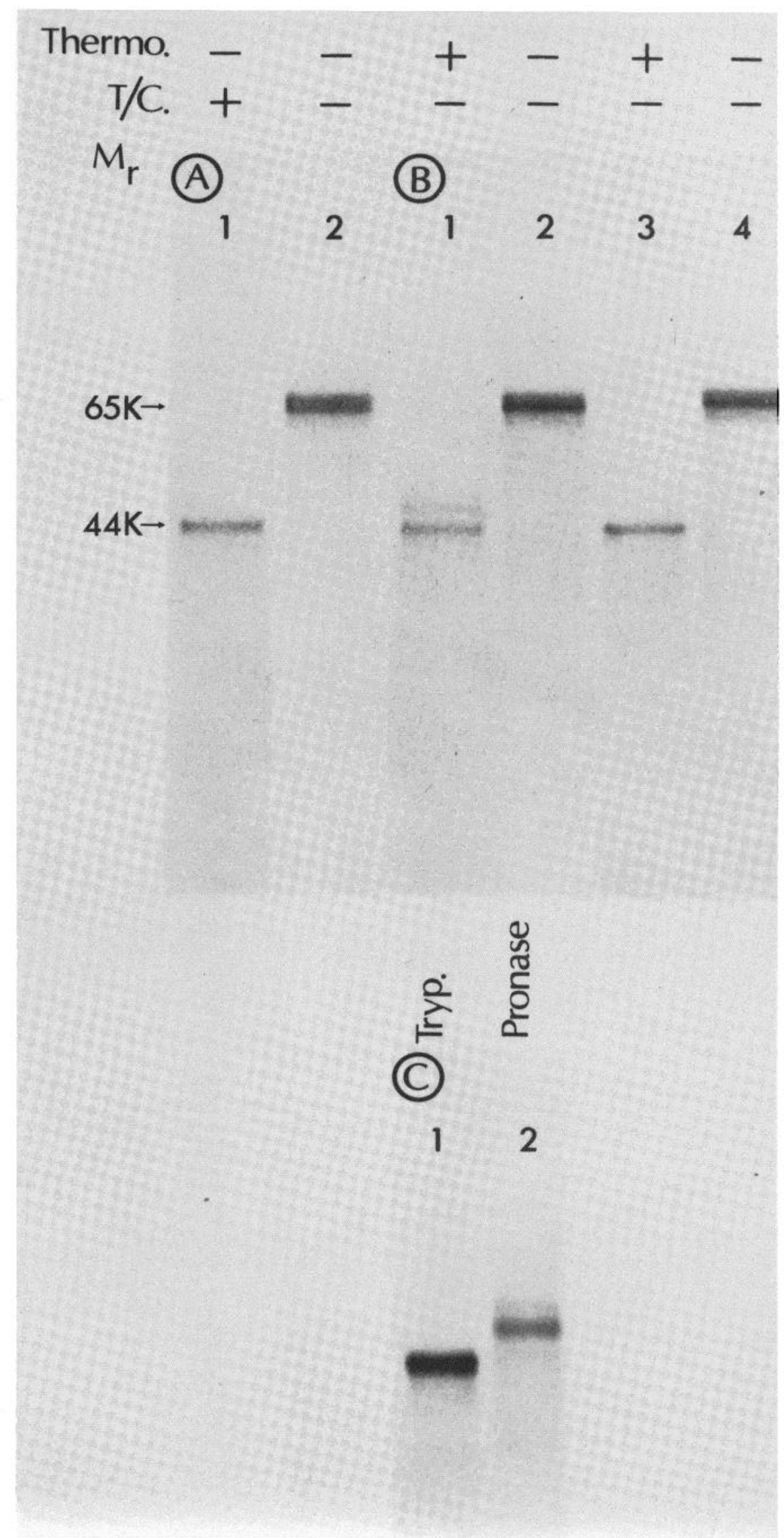

Figure 1. Proteolytic cleavage of AChR- $\delta$ from the cytoplasmic surface yields a single, membrane-protected fragment. $A$ and $B$, Translation reaction mixtures $(50 \mu \mathrm{l})$ containing $\mathrm{RM}$ were incubated post-translationally with $(+)$ or without $(-) 300 \mu \mathrm{g} / \mathrm{ml}$ of thermolysin (Thermo.) or $300 \mu \mathrm{g} / \mathrm{ml}$ each of trypsin plus chymotrypsin $(T / C)$. Incubation was for $60 \mathrm{~min}$ at $0^{\circ} \mathrm{C}(A)$, or at $26^{\circ} \mathrm{C}(B)$ for $1 \mathrm{hr}$ (lanes 1 and 2) or $5 \mathrm{hr}$ (lanes 3 and 4 ) in the presence of $5 \mathrm{mM} \mathrm{CaCl}_{2}$. Reactions were terminated with 1000 units/ml of Trasylol $(A)$, or $10 \mathrm{mM}$ EDTA $(B)$. SDS-PAGE was performed on an $18 \%(\mathrm{w} / \mathrm{v})$ polyacrylamide gel containing $6 \mathrm{M}$ urea. $C$, As above, except that digestions were perfor 2 ), for $1 \mathrm{hr}$ at $0^{\circ} \mathrm{C}$. PAGE was carried out on a $10 \%$ jolyacrrolamide gel. 


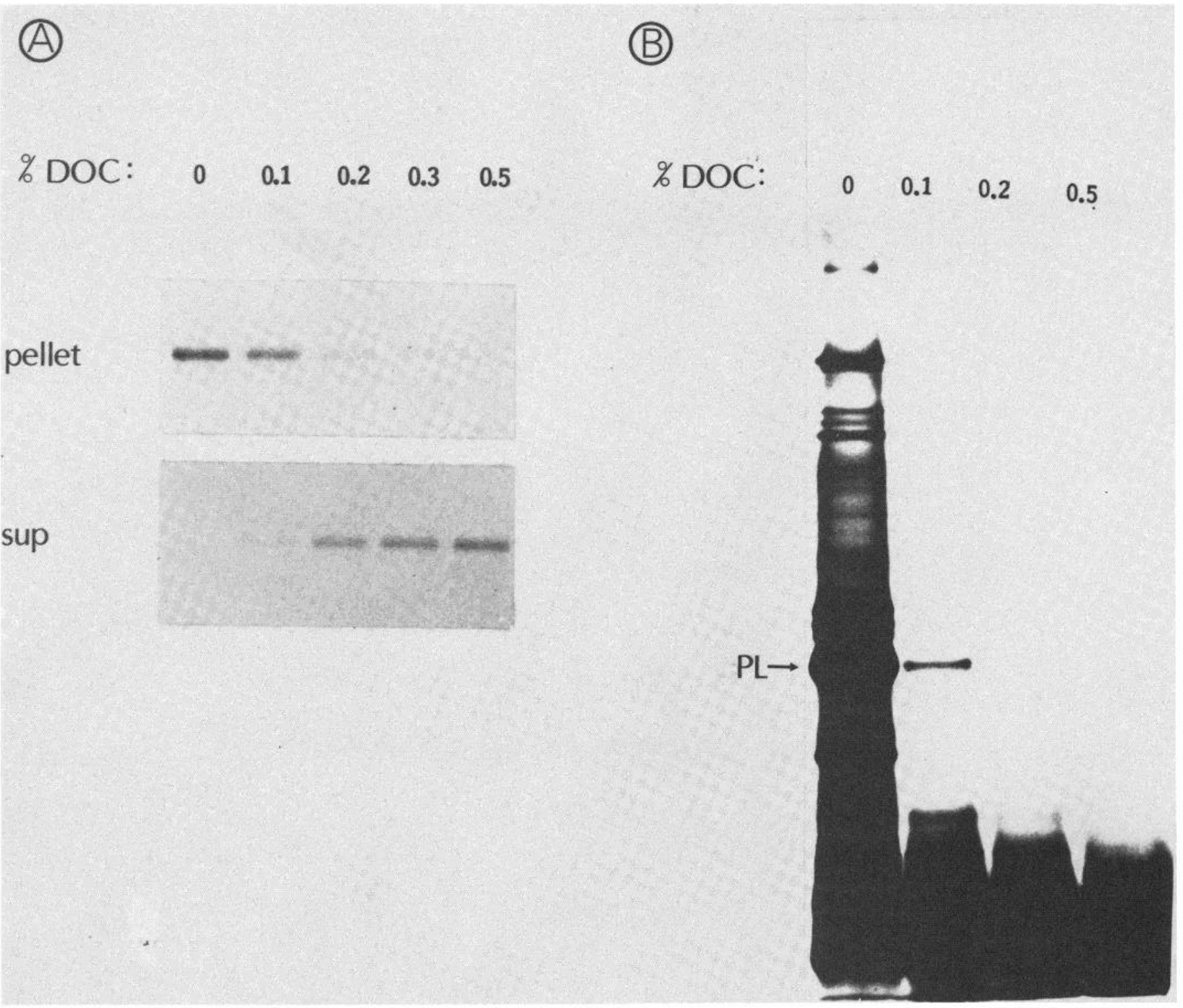

Figure 2. Permeabilization of RMs to trypsin without solubilization of AChR- $\delta . A, \mathrm{RMs}$ were reisolated from translation reaction mixtures as described under "Materials and Methods" and diluted to $132 \mu \mathrm{l}$ with buffer. DOC solubilization was performed by addition of $0.25 \mathrm{vol}(32 \mu \mathrm{l})$ of 5 times concentrated detergent solution. This brought the final RM concentration to $\sim 7 \mathrm{~A}_{280}$ units $/ \mathrm{ml}$. After a 30 -min incubation on ice, RM residues were pelleted through a 100 - $\mu \mathrm{l}$ cushion of $0.5 \mathrm{M}$ sucrose at $100,000 \times g_{\text {av }}$ for $10 \mathrm{~min}$ in an Airfuge. The supernatant (sup) and pellet fractions were recovered and immunoprecipitated as described in the legend to Figure $6 . B$, Translation reaction mixtures $(25 \mu \mathrm{l})$ containing $4 \mathrm{~A}_{260} \mathrm{units} / \mathrm{ml}$ of bovine pituitary total RNA were incubated in the presence of RM. Membrane reisolation and detergent solubilization were performed as in $A$. Samples were then made $50 \mu \mathrm{g} / \mathrm{ml}$ in trypsin and incubated for $60 \mathrm{~min}$ at $0^{\circ} \mathrm{C}$. Reactions were terminated by addition of Trasylol and samples were processed for electrophoresis without prior immunoprecipitation. $P L$ indicates prolactin. The autoradiograph was overexposed to show that a small amount $(<10 \%)$ of PL was resistant to trypsin at $0.1 \%$ DOC but was completely degraded at $0.2 \%$ DOC. Assays like those in $A$ showed that $0.1 \%$ DOC released about $60 \%$ of the PL from the RM; this cannot account for the $>90 \%$ of PL that was degraded and, therefore, at $0.1 \%$ DOC, trypsin must have entered the vesicles. The higher molecular weight bands in lane 0 are "readout" products of the pancreas RM-associated mRNAs (RMs were not nuclease-digested).

to probe the cytoplasmic domains of AChR- $\delta$ integrated into microsomal membranes. The membranes are protease-impermeant (Blobel and Dobberstein, 1975) vesicles of uniform sidedness (Jackson and Blobel, 1977), the cytoplasmic surface being exposed to the aqueous medium. They are therefore topologically inside-out with respect to Torpedo plasma membrane vesicles (Sobel et al., 1977; Hartig and Raftery, 1979; Klymkowsky et al., 1980; Saint-John et al., 1982). Proteolysis of transmembrane proteins integrated into these microsomal vesicles therefore occurs exclusively at the cytoplasmic pole of these proteins.

Membrane-integrated AChR- $\delta$ digested with $50 \mu \mathrm{g} / \mathrm{ml}$ of trypsin was cleaved into two major fragments immu- 


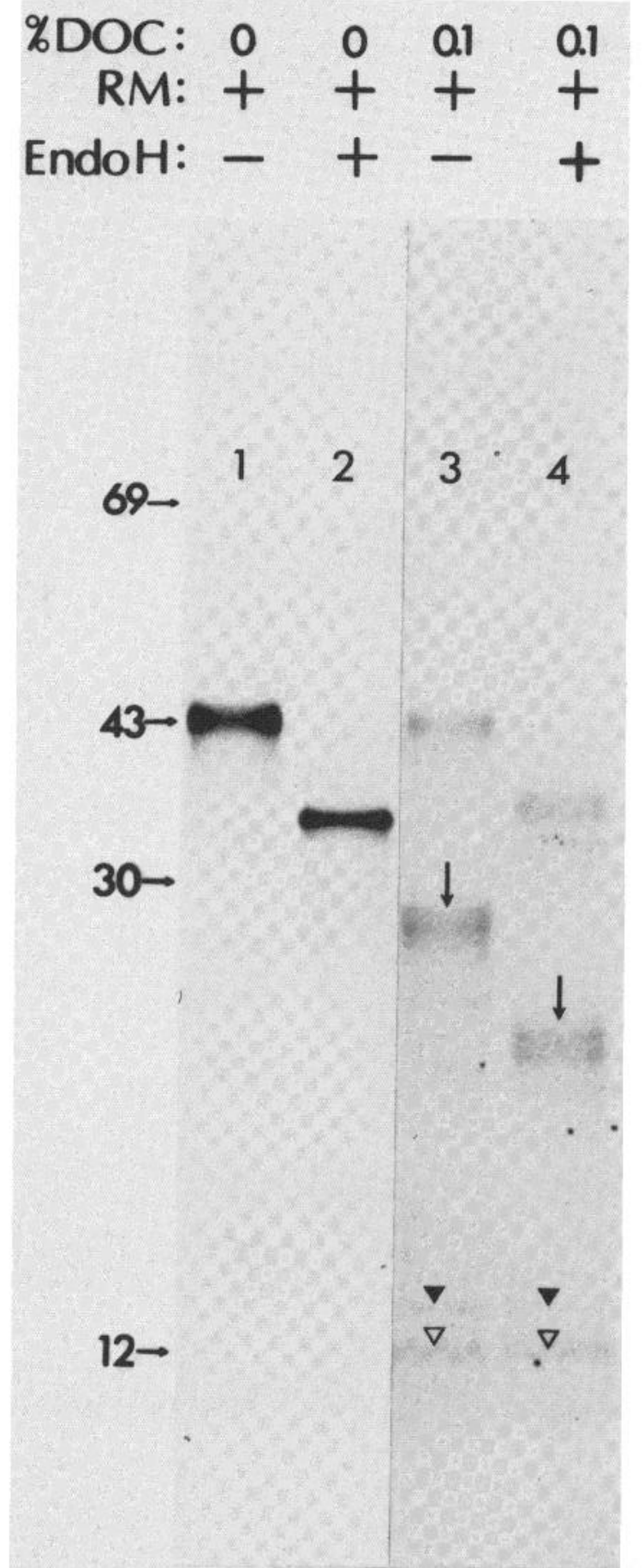

Figure 3. Tryptic cleavage of the 44-kd fragment from the luminal surface of the membrane. RMs were reisolated from $100-\mu \mathrm{l}$ translation reaction mixtures and diluted as described in Figure 2. Digestion with $50 \mu \mathrm{g} / \mathrm{ml}$ of trypsin was performed on all samples, in either the absence (lanes 1 and 2) or presence (lanes 3 and 4 ) of $0.1 \%$ DOC. Subsequent to immunoprecipitation the samples were incubated either with (lanes 2 and 4) or without (lanes 1 and 3) Endo $\mathrm{H}$ as previously described (Anderson et al., 1982). Lanes 3 and 4 are from a different gel than lanes 1 and 2, and were aligned using molecular weight markers. The poor recovery of the 26 -kd material can be ascribed to the low titers of antibodies to this domain in the pAb serum (see also "Discussion"). Lanes 3 and 4 represent a longer exposure than do lanes 1 and 2 . Thus, the 12 -kd band visible in the former (open arrowheads) is not apparent in the

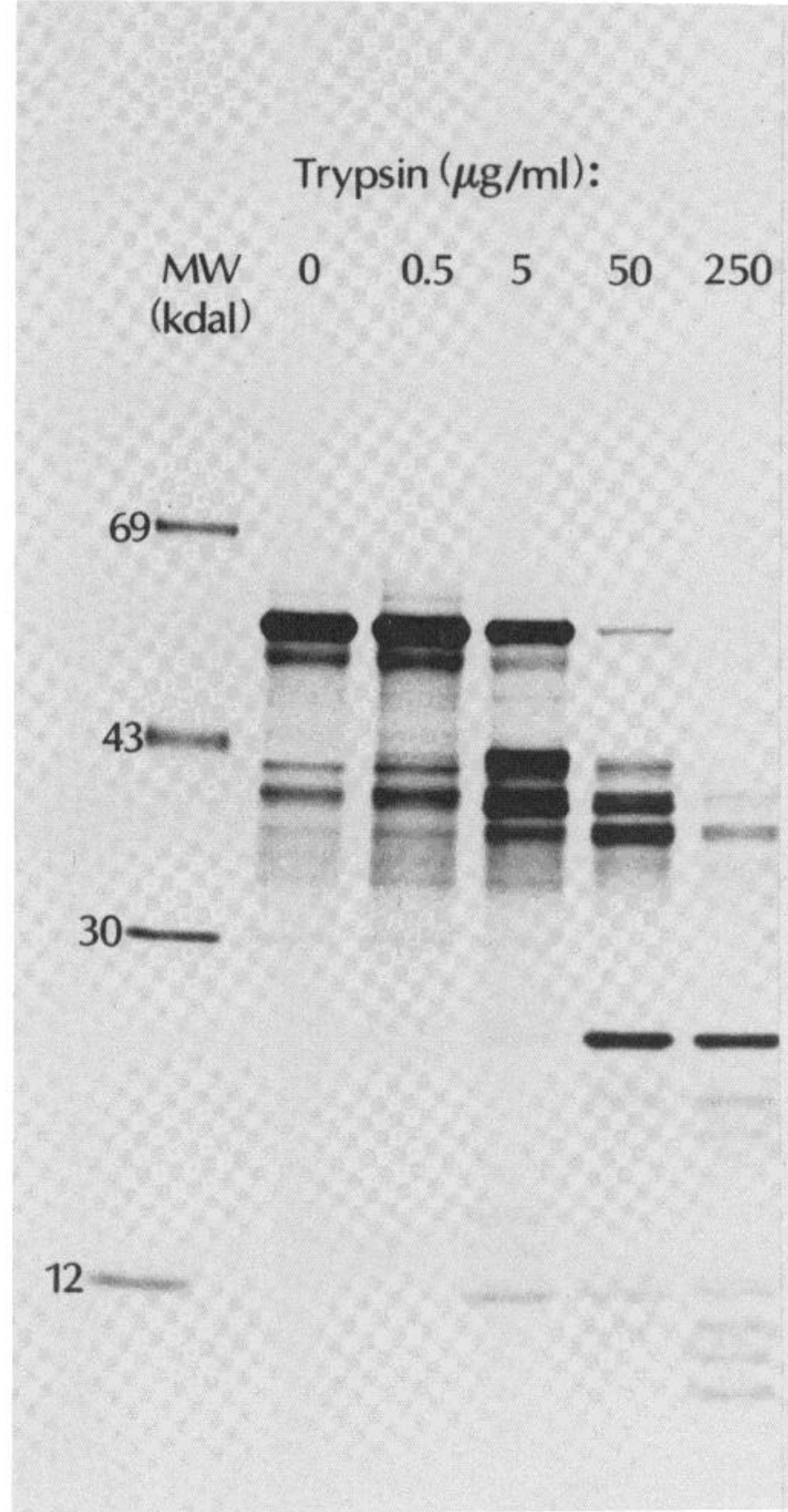

Figure 4. Fragmentation of pre-AChR- $\delta$ by trypsin. Aliquots $(50 \mu \mathrm{l})$ of in vitro translation reaction mixtures (lacking $\mathrm{RM}$ ) were treated with the indicated concentrations of TPCK-tryp$\sin$ for $60 \mathrm{~min}$ at $0^{\circ} \mathrm{C}$. The samples were then denatured in SDS and immunoprecipitated. $M W$ indicates molecular weight markers in kilodaltons. SDS-PAGE was performed on a 7.5 to $15 \%$ linear gradient gel. The fluorograph has been overexposed so that the more complex pattern of fragments produced at higher trypsin concentrations can be visualized. As a result, several minor bands are visible in the " 0 trypsin" sample in addition to the major $M_{\mathrm{r}}=59,000$ species. These bands most likely represent minor contaminants in the immunoprecipitates; however, their intensity is too low for them to contribute significantly to the pattern of major tryptic fragments.

latter. This band was visible in longer exposures of lanes 1 and 2 , although material in the 26 -kd region was never detected. SDS-PAGE was performed on a 10 to $15 \%$ linear gradient polyacrylamide gel. 
noprecipitable with our polyclonal antibody (Fig. $5 A$, $p A b-\delta,+$ ): the 44-kd glycosylated domain (see also Fig. 1 ), and a new, smaller species of approximately $12 \mathrm{kd}$ (Fig. 5A, downward arrow). At higher trypsin concentrations this fragment can be converted to a slightly smaller species that can be seen faintly in the same lane (Fig. $5 A$, upward arrow.) Because of the topology of these vesicles this fragment must necessarily derive from the cytoplasmic domain of the protein.

We next sought to determine whether this 12 -kd fragment of AChR- $\delta$ constituted a water-soluble cytoplasmic domain. To this end, we fractionated translation reaction mixtures containing trypsinized AChR- $\delta$ in RM by centrifugation, prior to immunoprecipitation. Using a monoclonal antibody (see below), we detected 60 to $70 \%$ of the $12-\mathrm{kd}$ fragment in a supernatant fraction at $\mathrm{pH} 10$ (Fig. 6A, lane $S$, arrow), indicating partial solubilization of this domain after cleavage. As expected, the complementary 44-kd domain, immunoprecipitated with the anti- $\delta \mathrm{pAb}$, was quantitatively recovered in the pellet fraction (Fig. $6 B$, lane $P$ ), indicating that the observed distribution of the 12 -kd fragment was not due to incom- plete sedimentation of the microsomal membranes. The 12 -kd fragment, in addition, constitutes one of the low molecular weight species (indicated by the open arrowhead in Fig. 3, lanes 3 and 4) produced after trypsin digestion in the presence of DOC (this was established using the monoclonal antibody, described in the following section). This is to be expected, as DOC allows simultaneous digestion at both the cytoplasmic and extracellular poles of $\mathrm{AChR}-\delta$.

Domain-specificity of monoclonal antibodies to AChR$\delta$. The preceding results indicated that cleavage of $\mathrm{AChR}$ $\delta$ with low concentrations of trypsin dissected the molecule into two immunoprecipitable domains: a 44-kd, membrane-integrated domain, and a partially soluble 12 $\mathrm{kd}$ fragment comprising a portion of an untranslocated domain. Both fragments reacted with our polyclonal anti- $\delta$ antibody. We next assayed the reactivity of these fragments toward various anti- $\delta \mathrm{mAbs}$, with a view toward establishing the domain specificities of these mAbs (Tzartos and Lindstrom, 1980).

When AChR- $\delta$ cleaved by $50 \mu \mathrm{g} / \mathrm{ml}$ of trypsin was immunoprecipitated after SDS denaturation with var-

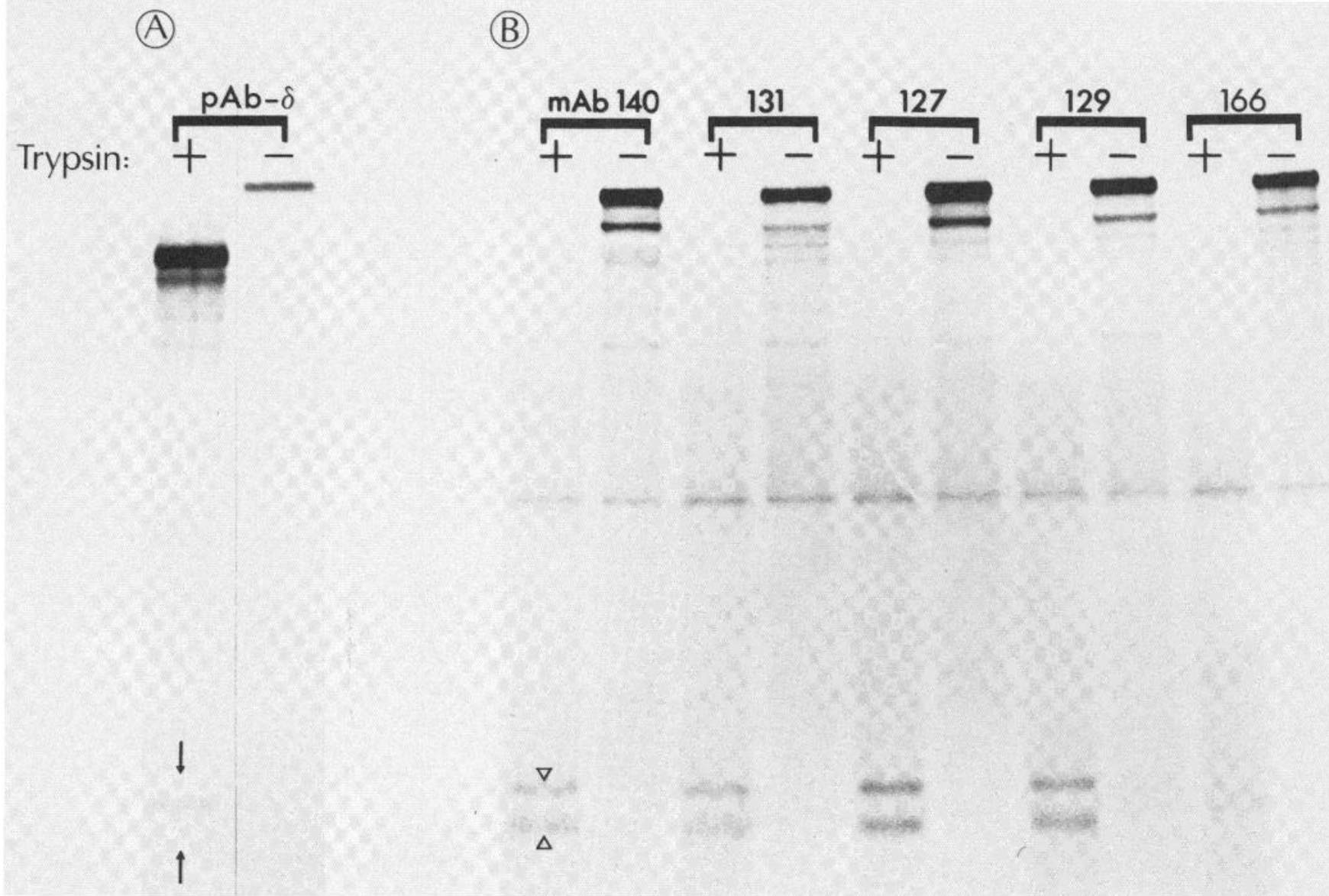

Figure 5. Identification of a cytoplasmic domain of AChR- $\delta$ and of mAbs directed against it. Translation reaction mixtures $(50 \mu \mathrm{l})$ containing $2 \mathrm{~A}_{280}$ units/ml of $\mathrm{RM}$ were incubated post-translationally either with $(+)$ or without (-) $50 \mu \mathrm{g} / \mathrm{ml}$ of TPCKtrypsin for $60 \mathrm{~min}$ at $0^{\circ} \mathrm{C}$. After SDS solubilization, samples were immunoprecipitated with either the polyclonal anti- $\delta$ antiserum $(p A b-\delta$; in $A)$ or with the indicated mAbs $(B)$. Double arrows indicate the two forms of the cytoplasmic fragment; as $A$ and $B$ are taken from different experiments, the relative proportion of these forms varies slightly. The minor bands seen below the 44 $\mathrm{kd}$ fragment in $A$ are derived from incompletely glycosylated but membrane-integrated forms of AChR- $\delta$, as previously shown (Anderson et al., 1982). The $M_{\mathrm{r}}=30,000$ band seen in all lanes of $B$ represents contamination by a major secretory protein (see also Fig. 6) of Torpedo. It is found only when goat anti-rat IgG-agarose is used as the immunoadsorbent, as was the case in $B$; in $A$, protein A-Sepharose was used and the band is absent. 


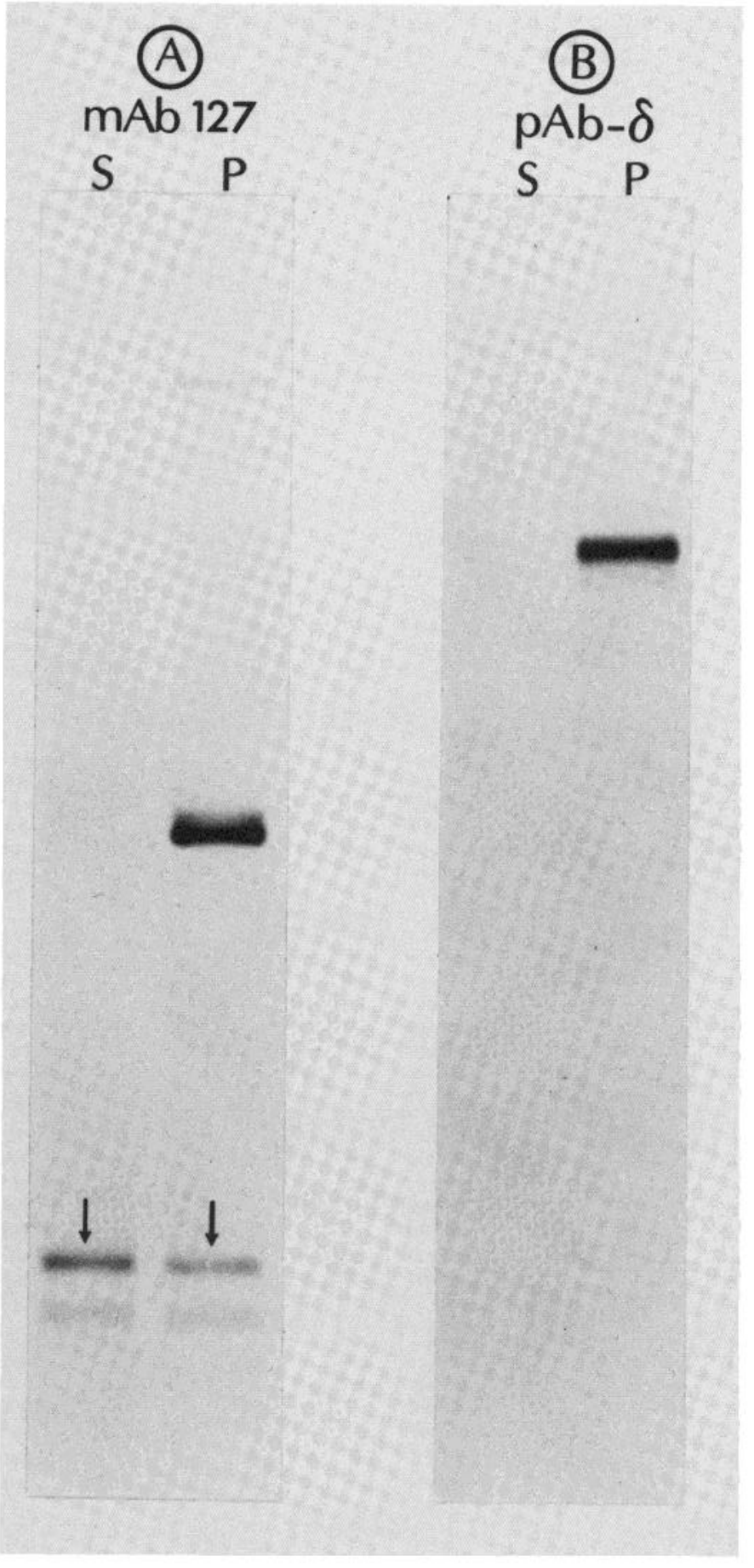

Figure 6. Solubility properties of 12 -kd AChR- $\delta$ fragment. Translation reaction mixtures containing $\mathrm{RM}$ were digested with either $50(A)$ or $300(B) \mu \mathrm{g} / \mathrm{ml}$ of trypsin. Subsequent to proteolysis, samples were adjusted to $\mathrm{pH} 10$ with $1 \mathrm{~N} \mathrm{NaOH}$ and incubated on ice for $30 \mathrm{~min}$, and the $\mathrm{RMs}$ then were pelleted in an Airfuge for $2 \mathrm{~min}$ at $100,000 \times g_{\text {av }}$. Immunoprecipitations were performed with the antibodies indicated above the lanes. The supernatant fractions $(S)$ were treated as usual; pellets $(P)$ were resuspended directly in $1 \%$ SDS and then diluted into immunoprecipitation buffer containing $100 \mu \mathrm{g} / \mathrm{ml}$ of bovine serum albumin as carrier. The $30-\mathrm{kd}$ contaminant pelleted as it is a secretory protein. ious anti- $\delta \mathrm{mAbs}$, a number of the antibodies were found to react with the 12 -kd fragment (Fig. $5 B$, numbers 140 , 131,127 , and 129). Based on the foregoing characterization of this fragment, these mAbs must all be specific to the cytoplasmic domain of $\delta$ subunit. Surprisingly, no mAbs were found to react with the complementary, 44$\mathrm{kd}$ fragment (Fig. $5 B$; data not shown.) This finding is consistent with an earlier observation (Froehner, 1981) that polyclonal anti- $\delta$ antisera contain very low titers of antibodies directed against extracellular domains of the subunits. Interestingly, a large number of anti- $\delta \mathrm{mAbs}$ were found which reacted with neither the 44-kd nor the 12 -kd fragments (Fig. $5 B$, lane $166+$ ), although they did recognize intact $\mathrm{AChR}-\delta$ (Fig. 5B, lane 166-). As the 44$\mathrm{kd}$ piece contains both extracellular- and membranespanning domains, mAb 166 must, by elimination, react with a determinant that resides in that portion of the trypsin-accessible $20-\mathrm{kd}$ domain that is not contained in the 12 -kd fragment. This determinant could, in principle, be present on either an untranslocated domain, or on a small, membrane-embedded domain that was proteolytically severed from the $44-\mathrm{kd}$ piece.

To determine whether mAb 166 could, in fact, recognize any proteolytic fragments of AChR- $\delta$, we used it to immunoprecipitate pre-AChR- $\delta$ (cf. Fig. 4) cleaved by 25 $\mu \mathrm{g} / \mathrm{ml}$ of trypsin (Fig. 7A, lane 2). MAb 166 did in fact react with a $42-\mathrm{kd}$ fragment of pre-AChR- $\delta$ (Fig. $7 A$, lane $3)$. This $42-\mathrm{kd}$ fragment is generated by trypsin simultaneously with the 12 -kd fragment (Fig. 4, lanes $0.5,5$ and 50 ) and is not recognized by mAbs to the 12 -kd fragment (Fig. 7A, lane 4). It therefore comprises a domain distinct from that containing the $12-\mathrm{kd}$ piece. The 42-kd fragment is further degraded, at higher trypsin concentrations, into fragments of 40 and $38 \mathrm{kd}$ (Fig. 4, lanes 5,50 and 250; Fig. 7A lane 2); these cleavages remove the determinant recognized by $\mathrm{mAb} 166$ (Fig. $7 A$, lane 3 ). The mAb 166 determinant is thus located at the carboxy terminal end of the 42 -kd fragment, on a piece not larger than $2 \mathrm{kd}$ (the next largest fragment, $40 \mathrm{kd}$, lacks the antigenic site). Such a small piece would not be resolved by our gel system, and it may not contain methionine, which is present on average only once every $6.5 \mathrm{kd}$ in AChR- $\delta$ (Lindstrom et al., 1979; Vandlen, et al., 1979). (MAb 166 does not bring down the equivalent of a 42-kd fragment in Fig. 5B (lane 166+), because at this trypsin concentration (Fig. 4, lane $50 \mu \mathrm{g} / \mathrm{ml}$ ) its immunoreactive 2 -kd carboxy terminal piece has already been removed.) The group of mAbs represented by 166 (and which included numbers 7 and 141) thus define a highly antigenic, presumably cytoplasmically exposed, domain of AChR- $\delta$.

Domain specificity of anti-AChR- $\beta$ mAbs. The preceding experiments indicated that proteolytic fragments of AChR- $\delta$, defined initially using a polyclonal antibody, could in turn be used to characterize anti- $\delta$ mAbs. The precedent set by these results implied that mAbs could be used to define and identify proteolytic fragments of AChR subunits, regardless of whether those fragments had previously been detected by polyclonal antibodies. To demonstrate this, we examined AChR- $\beta$. In that case, polyclonal anti- $\beta$ antibodies had previously defined a 37- 


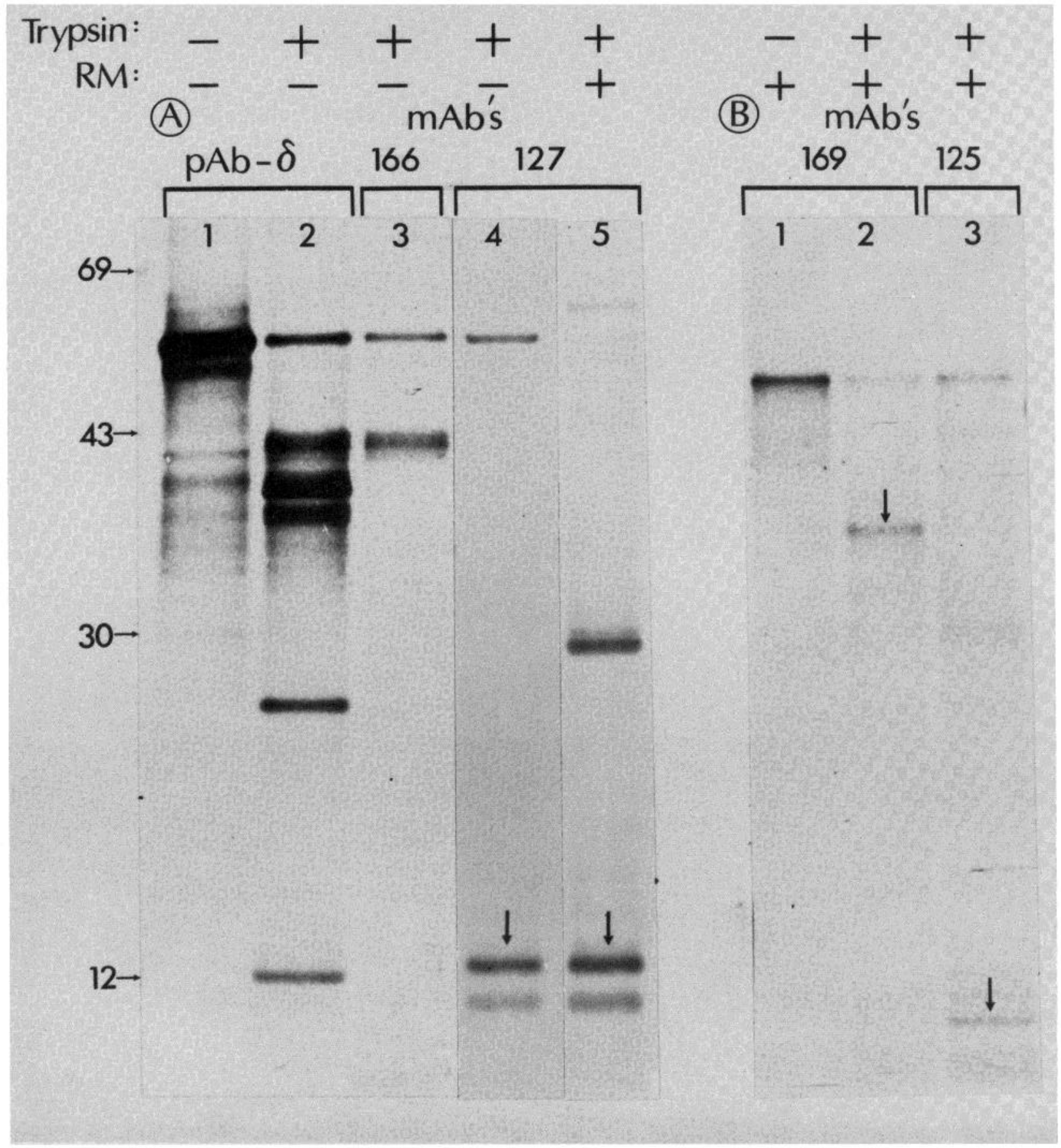

Figure 7. Further characterization of anti-AChR mAbs. A, Translation reaction mixtures $(50 \mu \mathrm{l})$ were incubated without (-) or with (+) RM and incubated post-translationally in either the absence $(-)$ or presence $(+)$ of $25 \mu \mathrm{g} / \mathrm{ml}$ of trypsin. Immunoprecipitation was carried out using the antibodies indicated above the brackets. Arrows in lanes 4 and 5 indicate the 12 $\mathrm{kd}$ fragment. These lanes are taken from a different gel than lane 2, and thus alignment of the 12-kd band in lane 2 with its counterpart in lanes 4 and 5 is imperfect. The calibration at the left indicates molecular weight standards, in kilodaltons. The $M_{\mathrm{r}}$ $=30,000$ contaminant is consistently absent from samples incubated without RM (cf. lanes 4 and 5 , also lane 3 with lanes 166 in Fig. 2). B, Topological characterization of anti-AChR- $\beta$ mAbs 169 and 125 . The Legend is as in A. Arrows indicate 38 -kd glycosylated fragment (lane 2) or 7-kd cytoplasmic fragment (lane 3). The $M_{r}=30,000$ contaminant is missing from these lanes as all samples were pretreated with goat anti-rat agarose, prior to addition of $\mathrm{mAb}$, to remove it.

kd, membrane-integrated domain (Anderson and Blobel, 1981) (analogous to the 44-kd domain of AChR- $\delta$ ), but had failed to detect a complementary, cytoplasmic domain. Such a domain was detectable, however, by $\mathrm{mAb}$
125 (Fig. 7B, lane 3), using the same procedure as in the case of the $\delta$ subunit (Fig. $5 B$ ). This cytoplasmic domain of AChR- $\beta$ has an apparent $M_{\mathrm{r}}$ of 7000 to 8000 and, like that of AChR- $\delta$, was partially soluble after cleavage and 
was readily degraded at higher trypsin concentrations (data not shown). Furthermore, anti- $\beta$ mAB 169 was found to react with the membrane-protected $37-\mathrm{kd}$ fragment (Fig. 7B, lane 2), in contrast to the case of $\mathrm{AChR}$ $\delta$, where no such $\mathrm{mAbs}$ were detectable. Taken together, these results indicate that post-translationally proteolyzed, in vitro integrated $\mathrm{AChR}$ subunits can successfully be used in a simple immunoprecipitation assay that simultaneously reveals the domain and topological specificities of anti-AChR mAbs.

\section{Discussion}

We have described the results of proteolytic dissection of an early biosynthetic form of AChR- $\delta$ subunit. This form of the subunit was obtained by in vitro translation of Torpedo electroplax mRNA, in the presence of heterologous microsomal membranes (Anderson and Blobel, 1981). Cleavage of $\delta$ at its cytoplasmic pole by low concentrations of trypsin dissected the molecule into two immunoprecipitable domains: a 44-kd, glycosylated domain (protected by the lipid bilayer from further degradation) and a $12-\mathrm{kd}$ fragment comprising a portion of the cytoplasmic domain. The 44-kd fragment was further cleaved into a $26-\mathrm{kd}$, glycosylated fragment, when the vesicles were rendered permeable to trypsin by low concentrations of deoxycholate. The $26-\mathrm{kd}$ piece thus comprises all, or part, of an extracellular domain of $\mathrm{ACh}-\delta$ (based on its content of core oligosaccharides). These data are summarized schematically in Figure 8.

Our results are in close accord with some of the data obtained by Wennogle et al. (1981) for $\delta$ in $\mathrm{NaOH}$. extracted Torpedo plasma membrane vesicles. In particular, they detected a small, cytoplasmic fragment of approximately $16 \mathrm{kd}$, that is likely to be related to the 12-kd species described here. In contrast to our findings, however, they were unable to subfragment the complementary 44-kd fragment into a $26-\mathrm{kd}$ species such as we obtained (Fig. 3). This difference, in the trypsin sensitivity of the extracellular domain of $\mathrm{AChR}-\delta$ in its rough endoplasmic reticulum and plasma membrane-associated forms, may be due to "masking" of certain tryptic cleavage sites during oligomeric assembly. Interestingly, a recent computer-generated model of the $\gamma$ subunit, derived from a cloned cDNA sequence, predicts a $25-\mathrm{kd}$ extracellular domain for this chain (Claudio et al., 1983). On the assumption (Conti-Tronconi and Raftery, 1982) that the primary structural homologies between the subunits (Raftery, et al., 1980) imply homology in, at least, the size of their extracellular domains, this prediction for $\gamma$ is in good agreement with our current results for the $\delta$ subunit. At their cytoplasmic poles, however, the subunits have been proposed to differ in proportion to their molecular weights (Conti-Tronconi and Raftery, 1982). We have obtained direct evidence for this, in the case of the cytoplasmic tryptic fragments recovered by mAbs 125 and 127 , for the $\beta$ and $\delta$ chains, respectively (see Fig. 7).

We were unable to directly identify the membraneembedded domains of AChR- $\delta$ in these experiments. These domains, together with the extracellular region, are contained within the 44-kd domain (cf. Fig. 1). As 26 $\mathrm{kd}$ of the 44-kd fragment are accounted for by the extracellular domain, the membrane-embedded domains could, by difference, comprise the remaining $18 \mathrm{kd}$. (The value of $18 \mathrm{kd}$ is calculated by subtraction; a discrete fragment of this size was not identified.) If all of this 18 $\mathrm{kd}$ was contained within the lipid bilayer, it would have to traverse the membrane several times. Indeed, the sequence-based model of AChR- $\gamma$ (Claudio et al., 1983) predicts up to four transmembrane helices for this subunit. If the proposed intersubunit structural homologies extend to the number of transbilayer segments, the computer prediction for $\gamma$ is again consistent with our estimate of the size of the membrane-embedded domain for $\delta$. These membrane-spanning segments might not be recovered as an intact, 18-kd fragment after trypsin digestion if they were severed from one another by cleavage at protease-sensitive sites on their (presumably) ex-

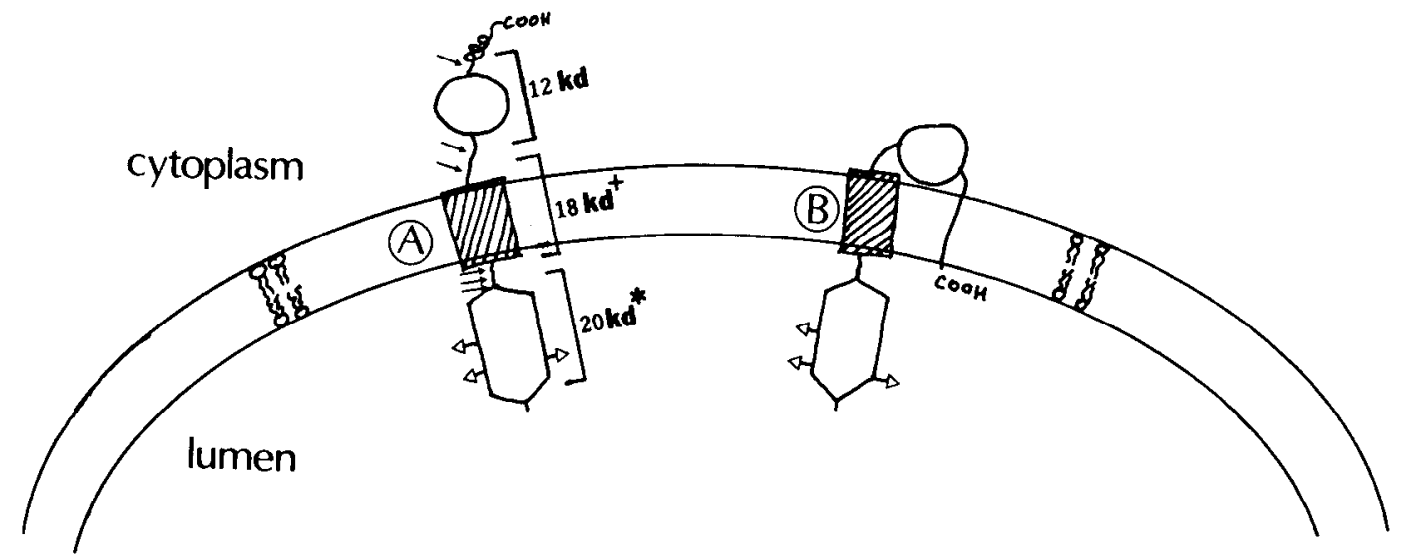

Figure 8. Schematic diagram summarizing data obtained for AChR- $\delta$. A, The circular domain on the cytoplasmic surface indicates the 12 -kd fragment identified in the present study. Additional polypeptide segments are drawn to show that, by difference, almost $20 \mathrm{kd}$ of $\mathrm{AChR}-\delta$ may be cytoplasmically exposed. + indicates that the hatched 18-kd transmembrane domain is calculated by subtraction and was not recovered as an intact fragment. The mass of the extracellular fragment $(*)$ is given minus the carbohydrate contribution. Arrows indicate the minimum number and probable location of tryptic sites needed to generate the fragment obtained. Open arrowheads on the extracellular domain indicate the core oligosaccharide groups. $B$ is drawn to indicate a variant of $A$, since we have no information as to the location of the carboxy terminus (Claudio et al., 1983 ). 
tracellularly exposed connecting loops. These membrane-spanning segments could conceivably comprise some of the small, Endo H-resistant bands seen after cleavage of AChR- $\delta$ simultaneously from the cytoplasmic and extracellular sides of the membrane (Fig. 3, solid arrowheads).

In the course of characterizing the proteolytic fragments of AChR- $\delta$, we developed an assay to define the domain specificities of monoclonal antibodies to the AChR subunits. Mabs specific to the cytoplasmic domains of both the $\delta$ and $\beta$ subunits were detected. Although no mAbs to the 44-kd fragment of AChR- $\delta$ were found, one $\mathrm{mAb}$ was identified which reacted with the analogous, 37-kd membrane-protected fragment of AChR- $\beta$ (number 169). This same antibody reacts with a $\sim 25-\mathrm{kd}$, glycosylated fragment of $\beta$, which is derived by trypsinization of $\mathrm{NaOH}$-extracted Torpedo vesicles (D. J. Anderson, unpublished observations). This indicates that the determinant recognized by mAb 169 lies on an extracellular domain of $\beta$, and that the size of this domain is similar to that shown here for $\delta$, and predicted for $\gamma$.

Interestingly, our assay detected a class of anti- $\delta \mathrm{mAbs}$ (e.g., numbers 7,141 and 166) that react with neither the $12-\mathrm{kd}$ nor the $44-\mathrm{kd}$ fragments. This is not unexpected, as the 12-kd fragment comprises only part of the $\sim 20$ $\mathrm{kd}$ which is removed from the subunit by proteolysis at its cytoplasmic pole. By elimination, therefore, the determinant recognized by this class of mAbs must reside on the missing 8-kd piece. Our failure to detect a discrete, 8-kd fragment with these antibodies could be due to a lack of methionine in the fragment, or most likely, to rapid proteolysis of the determinant. (This latter interpretation is supported by our experiments using preAChR- $\delta$ (cf. Figs. 7 and 4).) At present, we cannot distinguish whether the $\mathrm{mAb} 166$ determinant is truly exposed to the cytoplasm or is embedded in the membrane as a small segment. We feel the former possibility is more likely, however, as our data suggest that the determinant is contained on a 2 - to 3 -kd segment that is flanked by lysine or arginine (i.e., charged) residues. Monoclonal antibodies characterized in this way may eventually prove useful in correlating specific functional perturbations of $\mathrm{AChR}$, caused by a particular mAb (and measured in an isolated membrane "patch-clamp" recording), with defined structural domains recognized by that mAb.

Note added in proof. While this paper was in review, Noda et al. published the complete amino acid sequence of the $\delta$ subunit (Noda et al., 1983a) and a secondary structure model based upon this sequence (Noda et al., 1983b). Their model is consistent with the data presented here. Specifically, the $26-k d$ glycosylated domain we described probably extends from Lys 46 to the cluster ArgArg-Lys at positions 222 to 224. Similarly, we reason that as the $12-\mathrm{kd}$ cytoplasmic fragment is soluble (see our Fig. 6), it cannot contain the transmembrane segment "M4," and therefore is suggested to span the region from Lys 333 to the three lysines at positions 431, 434, and 436. By elimination, this would place the mAb 166 determinant in the stretch extending from Arg 315 to Lys 333.
Also while this paper was in press, a study by Gullick and Lindstrom (1983) appeared. It contains more extensive characterizations of the mAbs used here and is a useful crossreference.

\section{References}

Anderson, D. J., and G. Blobel (1981) In vitro synthesis, glycosylation and membrane insertion of the four subunits of Torpedo acetylcholine receptor. Proc. Natl. Acad. Sci. U. S. A. 78: 5598-5602.

Anderson, D. J., P. Walter, and G. Blobel (1982) Signal recognition protein is required for the integration of acetylcholine receptor $\delta$ subunit, a transmembrane glycoprotein, into the endoplasmic reticulum membrane. J. Cell Biol. 93: 501-506.

Blobel, G., and D. Dobberstein (1975) Transfer of proteins across membranes. II. Reconstitution of functional rough microsomes from heterologous components. J. Cell Biol. 67: 852-862.

Braell, W., and H. F. Lodish (1982) The erythrocyte anion transport protein is co-translationally inserted into microsomes. Cell 28: 23-31.

Claudio, T., M. Ballivet, J. Patrick, and S. Heinemann (1983) Nucleotide and deduced amino acid sequences of Torpedo acetylcholine receptor gamma subunit. Proc. Natl. Acad. Sci. U. S. A. 80: 1111-1115.

Conti-Tronconi, B. M., and M. A. Raftery (1982) The nicotinic cholinergic receptor: Correlation of molecular structure with functional properties. Annu. Rev. Biochem. 51: 491-530.

Froehner, S. C. (1981) Identification of exposed and buried determinants of the membrane-bound acetylcholine receptor from Torpedo californica. Biochemistry 20: 4905-4915.

Goldman, B. M., and G. Blobel (1981) In vitro biosynthesis, core glycosylation and membrane integration of opsin. J. Cell Biol. 90: 236-242.

Gullick and Lindstrom (1983) Biochemistry, in press.

Hartig, P. R., and M. A. Raftery (1979) Preparation of right side-out, acetylcholine receptor enriched intact vesicles from Torpedo californica electroplaque membranes. Biochemistry 18: $1146-1150$.

Jackson, R. C., and G. Blobel (1977) Post-translational cleavage of presecretory proteins with an extract of rough microsomes from dog pancreas containing signal peptidase activity. Proc. Natl. Acad. Sci. U. S. A. 74: 5598-5602.

Karlin, A. (1981) Molecular properties of nicotinic acetylcholine receptors. In The Cell Surface and Neuronal Function, C. W. Cotman, G. Poste, and G. L. Nicolson, eds., pp. 191260, Elsevier/North Holland Biomedical Press, New York.

Karlin, A., and D. Cowburn (1973) The affinity labeling of partially purified acetylcholine receptor from electric tissue of Electrophorus. Proc. Natl. Acad. Sci. U. S. A. 70: 36363640 .

Katz, F. N., J. E. Rothman, V. R. Lingappa, G. Blobel, and H. F. Lodish (1977) Membrane assembly in vitro: Synthesis, glycosylation and asymmetric insertion of a transmembrane protein. Proc. Natl. Acad. Sci. U. S. A. 74: 3278-3282.

Kistler, J., R. M. Stroud, M. W. Klymkowsky, R. A. Lalancette, and R. H. Fairclough (1982) Structure and function of an acetylcholine receptor. Biophys. J. 37: 371-383.

Klymkowsky, M. W., J. E. Heuser, and R. M. Stroud (1980) Protease effects on the structure of acetylcholine receptor membranes from Torpedo californica. J. Cell Biol. 85: 823838.

Kreibich, G., P. Debey, and D. D. Sabatini (1973) Selective release of content from microsomal vesicles without membrane disassembly. I. Permeability changes induced by low detergent concentrations. J. Cell Biol. 58: 436-462.

Lindstrom, J., J. Merlie, and G. Yogeeswaran (1979) Biochem- 
ical properties of acetylcholine receptor subunits from Torpedo californica. Biochemistry 18: 4465-4469.

Noda, M., H. Takahashi, T. Tanabi, M. Toyosato, S. Kikyotani, T. Hirose, N. Asai, H. Takashima, S. Inayama, T. Miyata, and S. Numa (1983a) Primary structures of $\beta$ and $\delta$ subunit precursors of Torpedo californica acetylcholine receptor deduced from cDNA sequences. Nature 301: 251-255.

Noda, M., H. Takahashi, T. Tanabi, M. Toyosato, S. Kikyotani, Y. Surutani, T. Hirose, H. Takashima, S. Inayama, T. Miyata, and S. Numa (1983b) Structural homology of Torpedo californica acetylcholine receptor subunits. Nature 302: 528 .

Oswald, R., A. Sobel, G. Waksman, B. Roques, and J. -P. Changeux (1980) Selective labelling by $\left[{ }^{3} \mathrm{H}\right]$ trimethisoquin azide of polypeptide chains present in acetylcholine receptorrich membranes from Torpedo marmorata. FEBS Lett. 111: 29-34.

Raftery, M. A., Hunkapiller, M. W., Strader, C. B. D. and Hood, L. E. (1980) Acetylcholine receptor: complex of homologous subunits. Science 208: 1454-1457.

Reynolds, J., and A. Karlin (1978) Molecular weight in detergent solution of acetylcholine receptor. Biochemistry 17: 2035-2038.

Saint-John, P. A., S. C. Froehner, P. A. Goodenough, and J. B. Cohen (1982) Nicotinic postsynaptic membranes from Torpedo: Sidedness, permeability to macromolecules, and topography of major polypeptides. J. Cell Biol. 92: 333-342.

Sobel, A., M. Weber, and J. -P. Changeux (1977) Large scale purification of the acetylcholine receptor protein in its mem- brane-bound and detergent extracted forms from Torpedo marmorata electric organ. Eur. J. Biochem. 80: 215-224.

Strader, C. D., and M. A. Raftery (1980) Topographic studies of Torpedo acetylcholine receptor subunits as a transmembrane complex. Proc. Natl. Acad. Sci. U. S. A. 77: 5807-5811.

Tzartos, S. J., and J. Lindstrom (1980) Monoclonal antibodies used to probe acetylcholine receptor structure: Localization of the main immunogenic region and detection of similarities between subunits. Proc. Natl. Acad. Sci. U. S. A. 77: 755759.

Vandlen, F. L., W. C. -S. Wu, J. C. Eisenach, and M. A. Raftery (1979) Studies of the composition of purified Torpedo californica acetylcholine receptor and of its subunits. Biochemistry 18: 1845-1854.

Weill, C. L., H. G. McNamee, and A. Karlin (1974) Affinity labeling of purified acetylcholine receptor from Torpedo californica. Biochem. Biophys. Res. Commun. 61: 997-1003.

Wennogle, L. P., and J. -P. Changeux (1980) Transmembrane orientation of proteins present in acetylcholine receptor-rich membranes from Torpedo marmorata studied by selective proteolysis. Eur. J. Biochem. 106: 381-393.

Wennogle, L. P., R. Oswald, T. Saitoh, and J. -P. Changeux (1981) Dissection of the 66,000 Dalton subunit of the acetylcholine receptor. Biochemistry 220: 2492-2497.

Wise, D. S., J. Wall, and A. Karlin (1981) Relative locations of the $\beta$ and $\delta$ chains of the acetylcholine receptor determined by electron microscopy of isolated receptor trimer. J. Biol. Chem. 256: 12624-12627. 